Effect of Fe doping on structure, charge ordering, magnetic and transport properties of $\mathrm{La}_{0.33} \mathrm{Ca}_{0.67} \mathrm{Mn}_{1-y} \mathrm{Fe}_{y} \mathrm{O}_{3}(0 \leq y \leq 0.06)$

This article has been downloaded from IOPscience. Please scroll down to see the full text article. 2006 J. Phys.: Condens. Matter 186729

(http://iopscience.iop.org/0953-8984/18/29/013)

View the table of contents for this issue, or go to the journal homepage for more

Download details:

IP Address: 82.151.111.197

The article was downloaded on 28/02/2013 at 11:10

Please note that terms and conditions apply. 


\title{
Effect of Fe doping on structure, charge ordering, magnetic and transport properties of $\mathrm{La}_{0.33} \mathrm{Ca}_{0.67} \mathrm{Mn}_{1-y} \mathrm{Fe}_{y} \mathrm{O}_{3}(0 \leqslant y \leqslant 0.06)$
}

\author{
T S Orlova ${ }^{1,2}$, J Y Laval ${ }^{1}$, P Monod ${ }^{1}$, J G Noudem ${ }^{3}$, V S Zahvalinskii ${ }^{4}$, \\ V S Vikhnin ${ }^{2}$ and Yu P Stepanov ${ }^{2}$ \\ ${ }^{1}$ Laboratoire de Physique du Solide, CNRS ESPCI, 10 rue Vauquelin, 75231 Paris cedex 05, \\ France \\ ${ }^{2}$ A F Ioffe Physico-Technical Institute, 26 Polytekhnicheskaya, St Petersburg 19402, Russia \\ ${ }^{3}$ Laboratoire de Cristallographie et Sciences des Materiaux, ISMRA, 6 Boulevard du Marechal \\ Juin, 14050 Caen Cedex, France \\ ${ }^{4}$ Belgorod State University, 85 Pobeda, 308015 Belgorod, Russia
}

Received 15 March 2006, in final form 15 May 2006

Published 30 June 2006

Online at stacks.iop.org/JPhysCM/18/6729

\begin{abstract}
The relationships between incommensurability, charge ordering and magnetic and electric transport properties were studied in $\mathrm{La}_{0.33} \mathrm{Ca}_{0.67} \mathrm{Mn}_{1-y} \mathrm{Fe}_{y} \mathrm{O}_{3}$ $(0 \leqslant y \leqslant 0.06)$ polycrystalline samples. Incommensurability and charge ordering were directly observed by electron diffraction versus temperature and lattice imaging at low temperature in transmission electron microscopy. Both the charge ordering and the ferromagnetic Curie-Weiss temperature linearly decrease with increasing $\mathrm{Fe}$ concentration $y$. A mean field analysis yields an antiferromagnetic exchange $J(\mathrm{Mn}-\mathrm{Fe})=-22 \mathrm{~K}$. The undoped samples showed predominantly a commensurate charge ordering superstructure with $\boldsymbol{q}$-vector $1 / 3 \boldsymbol{a}^{*}$ over the whole temperature range $<200 \mathrm{~K}$. For doped compositions, the $\boldsymbol{q}$-vector $(1 / 3-\varepsilon) \boldsymbol{a}^{*}$ is reduced by $12-15 \%$ for $5 \%$ Fe doping, as compared to the undoped composition. The low-temperature superstructure of the Fe-doped manganite does not correspond exactly to the threefold cell with lattice parameters $3 a, b, c$, and defects inducing shifts along the $a$ direction create a disturbance in long-range charge ordering. Jahn-Teller effects on $\mathrm{Mn}^{3+}$ centres are proposed to play a crucial part in the superstructure formation as well as in the suppression of charge ordering temperature by doping with non-JahnTeller impurity $\mathrm{Fe}^{3+}$ on $\mathrm{Mn}^{3+}$ sites.
\end{abstract}

\section{Introduction}

Since the discovery of colossal magnetoresistance (CMR) properties in $\operatorname{Ln}_{1-x} \mathrm{Ae}_{x} \mathrm{MnO}_{3}(\mathrm{Ln}$, lanthanide; Ae, alkaline earth) many studies have been carried out to elucidate the many 
interesting properties of these perovskites (see, for example, reviews [1-3]). It was found that $x$ could vary in a wide range, $0 \leqslant x \leqslant 1$, and consequently magnetic and electric transport properties could change deeply, since the system undergoes several phase transitions with different types of magnetic, electron and structure ordering. Most publications are devoted to $\mathrm{Ln}_{1-x} \mathrm{Ae}_{x} \mathrm{MnO}_{3}$ compounds $(0<x<0.5)$ with hole-type carriers. These compounds exhibit CMR: sample resistivity drops drastically under applied magnetic field near Curie temperature $T_{\mathrm{C}}$. The application of a magnetic field drives a phase transition from a paramagnetic insulator to a ferromagnetic metal in these manganites. The simultaneous presence of $\mathrm{Mn}^{3+}$ and $\mathrm{Mn}^{4+}$ ions in these compounds leads to the double exchange (DE) mechanism for the $\mathrm{Mn}^{3+}-\mathrm{O}-$ $\mathrm{Mn}^{4+}$ coupling, which can explain the appearance of the ferromagnetism as well as the phase transition [4]. In addition to DE, a specific role of the Jahn-Teller distortion around the $\mathrm{Mn}^{3+}$ ion is pointed out in several reports (for example, in [5]). The importance of phase separation indicating magnetically inhomogeneous structure at a microscopic level is also stressed in some other papers $[6,7]$.

The other part of the phase diagram of $\mathrm{Ln}_{1-x} \mathrm{Ae}_{x} \mathrm{MnO}_{3}$ with $x>0.5$ corresponds to compounds which exhibit charge ordering $(\mathrm{CO})$ below charge ordering temperature $T_{\mathrm{co}}$ [8-10]. First the possibility of $\mathrm{CO}$ was discussed by Goodenough [8] in connection with data obtained from observation by neutron diffraction [11] of superstructural peaks which are not of magnetic nature. The superstructural modulation is due to the development of a Jahn-Teller distortion of the $\mathrm{Mn}^{3+} \mathrm{O}_{6}$ octahedra: so, $\mathrm{d}\left(z^{2}\right)\left(\mathrm{Mn}^{3+}\right)$ orbitals are oriented perpendicular to the $c$-axis and form a series of ordered (zigzag) chains within the $(a-b)$ basal plane. This lattice distortion can be perfectly detected in transmission electron microscopy (TEM) observations made along the $c$-axis. For example, in the LaCaMnO system charge ordering stripes were observed in high resolution lattice images (HREM) by Chen et al [12-14]. However, the obtained HREM images did not display details of structural ordering due to mechanical instability of the samples at low $T$ in the microscope.

The charge ordering phenomenon in $\mathrm{Ln}_{0.5} \mathrm{Ae}_{0.5} \mathrm{MnO}_{3}$ manganites has been the subject of a considerable number of papers over the past few years [7, 15-19]. It has been shown that in these compositions there is an interplay between magnetism and transport properties through spin, charge and orbital ordering which is still not well understood. This composition presents a specific case since there is a competition between two types of magnetic ordering. Effectively, both ferromagnetic metallic and antiferromagnetic insulating ordering are observed in a small range around $x=0.5\left(\mathrm{Mn}^{3+} / \mathrm{Mn}^{4+}=1\right)$. However, these compositions are very sensitive to oxygenation value [16] and small changes in oxygen content could result in a shift from the hole doping part of the phase diagram to the electron doping or vice versa. It should be noted that the compositions with $x>0.5$ are less sensitive to the level of oxygenation because they are located farther from the 'border' of the phase diagram, but they are less studied.

The charge ordering mechanism is not sufficiently understood because it brings an interplay between $\mathrm{Mn}^{3+}: \mathrm{Mn}^{4+}$ ions, orbital and spin ordering. Doping by impurity elements appears to be very helpful for a better understanding of the $\mathrm{CO}$ phenomenon. So, doping by a small amount of different elements on Mn sites can lead to drastic changes in the physical properties of manganites, similarly to an applied magnetic field or pressure for instance. All these phenomena seem to have a common explanation, and the study of Mn site doping may play a key role in their understanding. For example, the insulator-metal transition in $\operatorname{Pr}_{0.5} \mathrm{Ca}_{0.5} \mathrm{Mn}_{1-y} \mathrm{Cr}_{y} \mathrm{O}_{3}$ was found in the absence of magnetic field [17, 18]. $\mathrm{Cr}$ doping in $\mathrm{La}_{0.3} \mathrm{Ca}_{0.7} \mathrm{Mn}_{0.8} \mathrm{Cr}_{0.2} \mathrm{O}_{3}$ composition results in the suppression of charge ordering and appearance of magnetoresistance [19]. This shows that it is possible to modify dramatically the phase diagram of the manganites by doping the Mn sites with a magnetic cation. 
Doping by $\mathrm{Fe}^{3+}$ also seems to be very appropriate since $\mathrm{Fe}^{3+}$ is magnetic, but does not give rise to the Jahn-Teller effect and unlike $\mathrm{Cr}$ does not participate in DE [20, 21]. The influence of $\mathrm{Fe}$ doping on magnetic and electrical properties was mainly studied for holedoped $\mathrm{La}_{1-x} \mathrm{Ca}_{x} \mathrm{Mn}_{1-y} \mathrm{Fe}_{y} \mathrm{O}_{3}(x=0.3$ and $x=0.25)$ systems [20, 22]. It was shown that in this part of the phase diagram Fe replaces $\mathrm{Mn}^{3+}$ and suppresses DE. In [20] a change in the character of electric resistivity behaviour of $\mathrm{La}_{0.75} \mathrm{Ca}_{0.25} \mathrm{Mn}_{1-y} \mathrm{Fe}_{y} \mathrm{O}_{3}$ was found for Fe concentration $y \sim 0.04$. It was explained by localization-delocalization transition of the quasi-particle excitation involved in the transport properties of this system. The data obtained on the behaviour of magnetization and electrical resistivity with temperature are rather contradictory for the $\mathrm{La}_{0.5} \mathrm{Ca}_{0.5} \mathrm{MnO}_{3}$ composition doped by $\mathrm{Fe}[21,23]$, but the compositions with $\mathrm{La} / \mathrm{Ca} \approx 1$ are very sensitive to oxygen content and a slight variation in oxygenation can strongly modify the physical properties [24], which most probably explains the contradictory data. For the composition with charge ordered antiferromagnetic ground state $(x>0.5)$, no systematic studies of Fe doping have been undertaken up to now. Furthermore, no LaCaMnO composition, with doping by $\mathrm{Fe}$, has been investigated yet from the structural point of view by TEM, although such experiments are conducive to the direct observation of the appearance of the superstructure and of its specificities.

In this work, we analysed the relationships between incommensurability, charge ordering and magnetic and transport properties in $\mathrm{La}_{0.33} \mathrm{Ca}_{0.67} \mathrm{Mn}_{1-y} \mathrm{Fe}_{y} \mathrm{O}_{3}(0 \leqslant y \leqslant 0.06)$ polycrystalline samples in a wide temperature range of $4.2-400 \mathrm{~K}$. The electron diffraction and microscopy experiments on charge ordering structures consisted in observing correlated changes in electron diffraction patterns and high resolution lattice images versus temperature, from room temperature down to $91 \mathrm{~K}$.

\section{Experimental details}

Polycrystalline samples were synthesized by solid state reaction. Starting materials of $\mathrm{La}_{2} \mathrm{O}_{3}$, $\mathrm{CaCO}_{3}, \mathrm{MnO}_{2}$ and $\mathrm{Fe}_{2} \mathrm{O}_{3}$ were mixed in stoichiometric proportions and heated twice in air at $900-1200^{\circ} \mathrm{C}$ for $24 \mathrm{~h}$ with intermediate grindings. Cold forming was performed under a uniaxial pressure of $30 \mathrm{MPa}$. Conventional annealing was conducted at $1300^{\circ} \mathrm{C}$ for $24 \mathrm{~h}$ in air. The prepared samples were characterized by powder x-ray diffraction (XRD) using a Philips automated $\mathrm{x}$-ray diffractometer with $\mathrm{Cu} \mathrm{K} \alpha$ radiation.

The electron diffraction (ED) versus temperature and lattice images in bright and dark field were carried out at room temperature and at low temperature down to $91 \mathrm{~K}$ with a JEOL $2010 \mathrm{~F}$ transmission electron microscope (TEM) operating at $200 \mathrm{kV}$ and equipped with a field emission gun (FEG-TEM) and a double tilt liquid $\mathrm{N}_{2}$ sample holder (tilt $\pm 15^{\circ}$ and $90 \mathrm{~K} \leqslant T \leqslant 300 \mathrm{~K}$ ). The images were processed with Digital Micrograph. In situ local compositional analysis was performed with a PGT energy selective X-ray Si-Li analyser (EDX) attached to the electron microscope.

Samples for electron microscopy were thinned by mechanical polishing followed by argon ion milling down to electron transparency $(\leqslant 50 \mathrm{~nm})$.

Resistance measurements were performed in the temperature range from 6 to $300 \mathrm{~K}$ in both cooling and heating regimes with a standard four-probe method on $2 \times 0.5 \times 8 \mathrm{~mm}^{3}$ samples.

The temperature dependence of the magnetization, $M(T)$, in the temperature range 4.2$400 \mathrm{~K}$ was measured with a commercial Quantum Design SQUID magnetometer after cooling the samples from room temperature down to $4.2 \mathrm{~K}$ in zero field (ZFC) or in different dc magnetic fields $H$ (FC).

The oxygen content in the studied samples was controlled by the iodometric titration method described, for example, in [25]. 
Table 1. The lattice parameters $a \approx b$ and $c$ in undoped and $5 \%$ Fe doped compounds.

\begin{tabular}{lcc}
\hline Composition & $a(\AA)$ & $c(\AA)$ \\
\hline $\mathrm{La}_{0.33} \mathrm{Ca}_{0.67} \mathrm{MnO}_{3}$ & 5.360 & 7.609 \\
$\mathrm{La}_{0.33} \mathrm{Ca}_{0.67} \mathrm{Mn}_{0.95} \mathrm{Fe}_{0.05} \mathrm{O}_{3}$ & 5.364 & 7.580 \\
\hline
\end{tabular}

Table 2. The results of iodometric titration studies.

\begin{tabular}{ll}
\hline Sample & $\begin{array}{l}\text { Oxygen content } \\
(3-\delta)\end{array}$ \\
\hline $\mathrm{La}_{0.33} \mathrm{Ca}_{0.67} \mathrm{MnO}_{3-\delta}$ & 2.966 \\
$\mathrm{La}_{0.33} \mathrm{Ca}_{0.67} \mathrm{Mn}_{0.95} \mathrm{Fe}_{0.04} \mathrm{O}_{3-\delta}$ & 2.963 \\
$\mathrm{La}_{0.33} \mathrm{Ca}_{0.67} \mathrm{Mn}_{0.95} \mathrm{Fe}_{0.05} \mathrm{O}_{3-\delta}$ & 2.964 \\
\hline
\end{tabular}

\section{Results and discussion}

\subsection{Characterization of samples}

The XRD patterns of all the studied compounds display a perovskite structure of the Pbnm space group with $a \approx b \approx \sqrt{2} a_{p}$ and $c \approx 2 a_{p}$ (where $a_{p} \sim 3.9 \AA$ is the lattice parameter of the simple perovskite structure [13]) and do not exhibit any other phase or any precipitate containing Fe. Analysis of the patterns for undoped and 5\% Fe doped samples gives lattice parameters shown in table 1 . We could not distinguish $a$ and $b$ parameters. Fe doping does not significantly change the lattice parameters. This can be explained by the substitution of $\mathrm{Mn}^{3+}$ by $\mathrm{Fe}^{3+}$ and by the fact that both ions have about the same size.

In addition, the chemical homogeneity of undoped and 5\% Fe doped samples was tested carefully by EDX with the very fine electron probe $(1 \mathrm{~nm})$ of our FEG-TEM. For this purpose, we analysed La:Ca:Mn ratio and distribution of Fe in different positions within a separate grain and among about 10-15 different grains.

In undoped composition, the chemical composition is very homogeneous within a separate grain, which corresponds to a variation smaller than $3 \%$ for $\mathrm{La} / \mathrm{Ca}$ ratio. However, chemical composition seems to be less homogeneous when going from one grain to another. In this case the $\mathrm{La} / \mathrm{Ca}$ ratio varies from 0.31 to 0.34 (0.33 on average). In the Fe-doped composition, variation of $\mathrm{La} / \mathrm{Ca}$ ratio within a separate grain does not exceed $5 \%$, but this ratio also changes in a wider range from 0.31 to 0.35 among different grains, giving the mean value about 0.33 . The concentration of Fe fits well under $5 \%$ from $(\mathrm{Mn}+\mathrm{Fe})$ content in all studied grains. We did not find any $\mathrm{Fe}$ segregation at grain boundaries. Since the spatial resolution in FEG-TEM $\mathrm{X}$-ray analysis is about $2 \mathrm{~nm}$, we can conclude that the distribution of $\mathrm{Fe}$ is homogeneous in the studied compounds. No secondary phases were observed in both compositions with TEM.

We achieved iodometric titration to check the oxygen content of the samples. As seen in table 2, the oxygen concentration is about the same in undoped and Fe doped compounds, though it differs slightly from the stoichiometric value: 3 .

\subsection{Magnetic properties}

The temperature dependence of the magnetization $M$ for the studied compositions is presented in figure 1(a) for magnetic field $H=1 \mathrm{~T}$ for field-cooled (FC) measurements. Figure 1(b) shows similar dependences for undoped and $5 \% \mathrm{Fe}$ doped compositions for zero-field-cooled (ZFC) and FC measurements in applied magnetic field $H=1 \mathrm{~T}$. In the undoped parent 


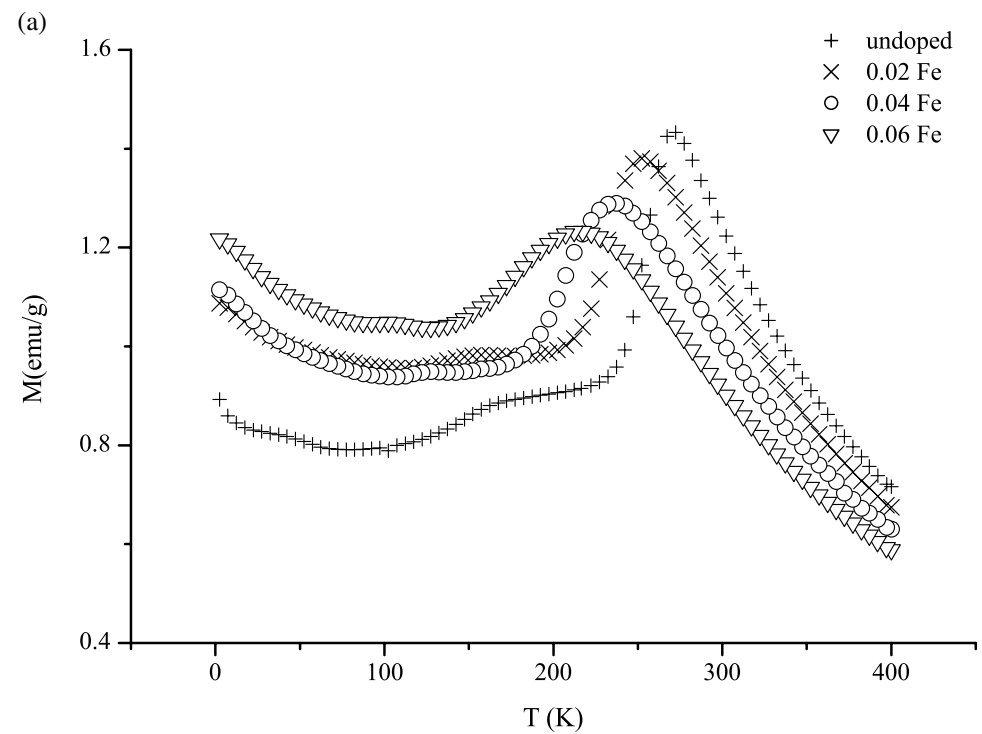

(b)

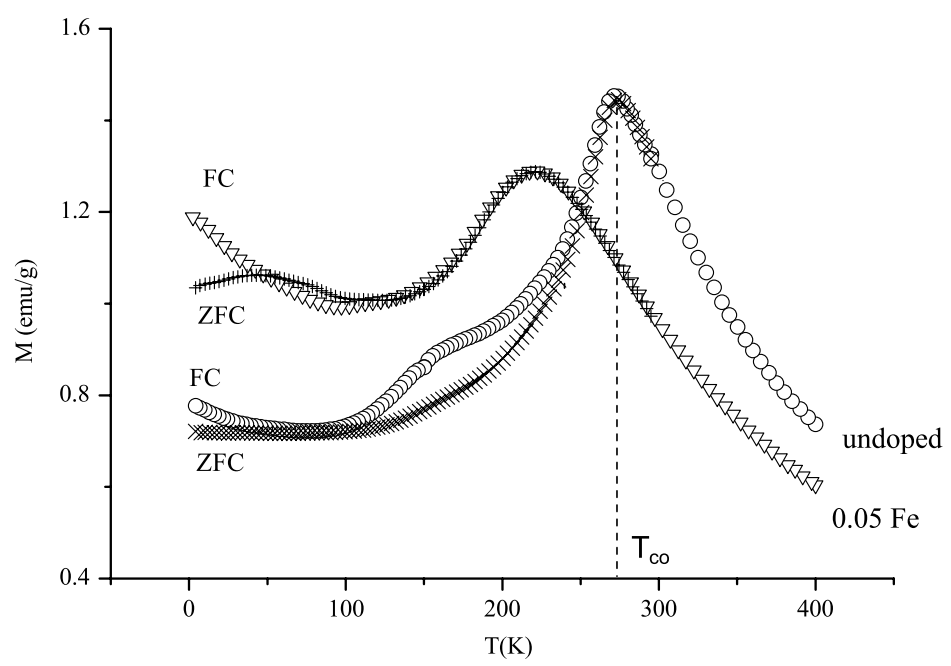

Figure 1. (a) Temperature dependence of magnetization for $\mathrm{La}_{0.33} \mathrm{Ca}_{0.67} \mathrm{Mn}_{1-y} \mathrm{Fe}_{y} \mathrm{O}_{3}$ with different Fe concentration $y$ obtained in field cooled measurements (FC) at magnetic field $H=1 \mathrm{~T}$. (b) Temperature dependence of zero-field cooling (ZFC) and field cooling (FC) magnetization of $\mathrm{La}_{0.33} \mathrm{Ca}_{0.67} \mathrm{MnO}_{3}$ and $\mathrm{La}_{0.33} \mathrm{Ca}_{0.67} \mathrm{Mn}_{0.95} \mathrm{Fe}_{0.05} \mathrm{O}_{3}$ in magnetic field $H=1 \mathrm{~T}$.

composition, several distinct features can be noted as being the onset for different magnetic behaviours: (i) the sharp drop in the magnetization just below $T_{\mathrm{co}}=272 \mathrm{~K}$, (ii) an irreversibility between the ZFC and FC magnetizations below $240 \mathrm{~K}$. The sharp change in magnetization at $T_{\text {co }}$ is associated with the transition from a paramagnetic state to a charge-ordering (CO) state as well known now for $\mathrm{La}_{1-x} \mathrm{Ca}_{x} \mathrm{MnO}_{3}$ with $x>0.5$. A charge ordering transition temperature $T_{\text {co }} \sim 260 \mathrm{~K}$ was reported for $\mathrm{La}_{0.3} \mathrm{Ca}_{0.7} \mathrm{MnO}_{3}$ [19]. Furthermore, for $\mathrm{La}_{0.33} \mathrm{Ca}_{0.67} \mathrm{MnO}_{3}$, a $T_{\text {co }} \sim 270 \mathrm{~K}$ was found from synchrotron x-ray and neutron powder measurements [26]. Thus, our data are in good quantitative agreement with literature data. 


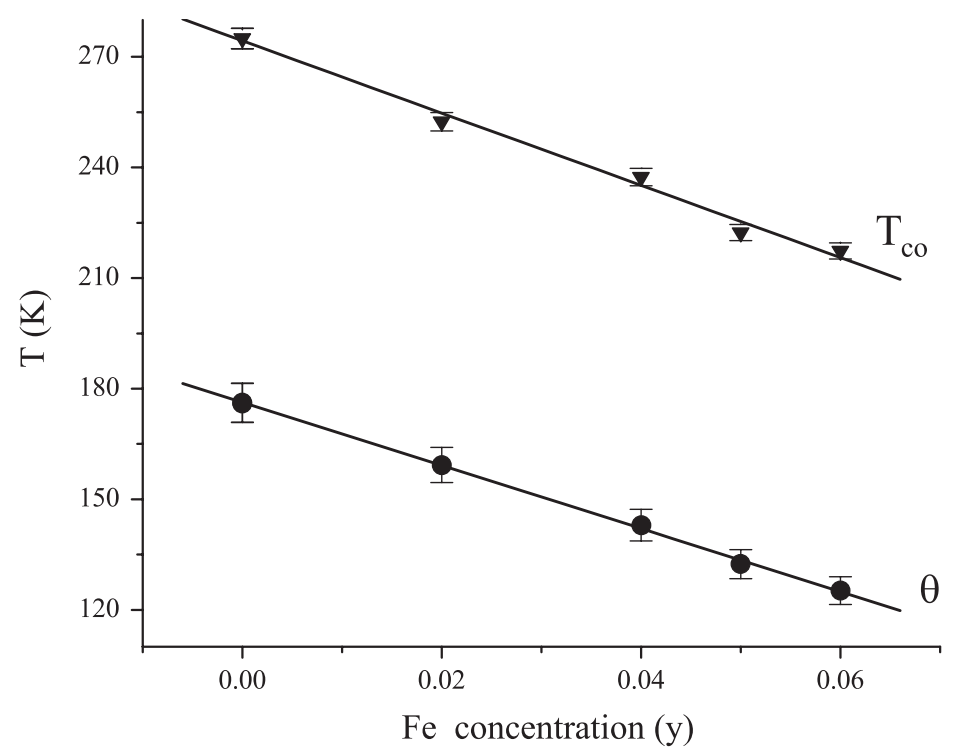

Figure 2. Characteristic temperatures versus Fe concentration $y: T_{\mathrm{co}}$-charge ordering transition temperature, $\theta$-Weiss temperature.

Previous phase diagrams $[9,27]$ of $\mathrm{La}_{1-x} \mathrm{Ca}_{x} \mathrm{MnO}_{3}$ system indicate that the ground state for $x=0.7$ composition is antiferromagnetic at low temperature with the Néel temperature $T_{\mathrm{N}} \sim 170 \mathrm{~K}$. We also observed a small, but noticeable change (so-called 'hump') in the slope of magnetization behaviour around $160 \mathrm{~K}$ even for the highest applied magnetic field, $1 \mathrm{~T}$. In [19] the authors connect it with the antiferromagnetic ordering.

The temperature dependences of the magnetization $M / H$ for $\mathrm{Fe}$ doped compositions (figure 1) demonstrate a similar behaviour as the undoped sample, but Fe doping leads to a pronounced shift of temperatures at which the character of specific magnetic behaviour changes. As will be shown below by direct registration of electron diffraction patterns in the transmission electron microscope, the characteristic temperature $T_{\text {co }}$ is the temperature of charge ordering transition in all studied compositions. Figure 2 shows $T_{\text {co }}$ versus Fe concentration $y$ dependence. Doping by Fe suppresses charge ordering: $T_{\text {co }}$ linearly decreases with the level of doping $y$ as

$$
T_{\mathrm{co}}(y)=T_{\mathrm{co}}(y=0)-A^{*} y
$$

for $0 \leqslant y \leqslant 0.06$, where $A^{*}=922 \pm 38 \mathrm{~K}, T_{\mathrm{co}}(y=0) \approx 272 \mathrm{~K}$.

The presence of a magnetic irreversibility and non-linear magnetic field dependence of the magnetization at $T<240 \mathrm{~K}$ was noted earlier for $\mathrm{La}_{0.3} \mathrm{Ca}_{0.7} \mathrm{MnO}_{3}$ [19]. It was explained by the presence of ferromagnetic clusters which nucleate at temperatures around $240 \mathrm{~K}$ and can co-exist with the antiferromagnetic state at lower temperature. The co-existence of small ferromagnetic clusters of Mn spins with an antiferromagnetic, charge-ordered structure at temperatures below $T_{\text {co }}$ was also reported in [28] for $\mathrm{La}_{1-x} \mathrm{Ca}_{x} \mathrm{MnO}_{3}(0.53<x<0.65)$.

To verify that the magnetic behaviour at $T<240 \mathrm{~K}$ is associated with the presence of a ferromagnetic component, we measured magnetization versus magnetic field $H$ behaviour. Magnetization loops with fields up to $5 \mathrm{~T}$ have been measured at 4.2, 90 and $150 \mathrm{~K}$ for undoped and 5\% Fe doped compositions. The obtained data are shown in figures 3(a) and (b). At $4.2 \mathrm{~K}$ the zero-field magnetization $\Delta m$ extrapolated from high-field slope in $M(H)$ dependence is quite similar for undoped and doped samples: $\Delta m=0.006-0.008 \mu_{\mathrm{B}} / \mathrm{f}$.u. 

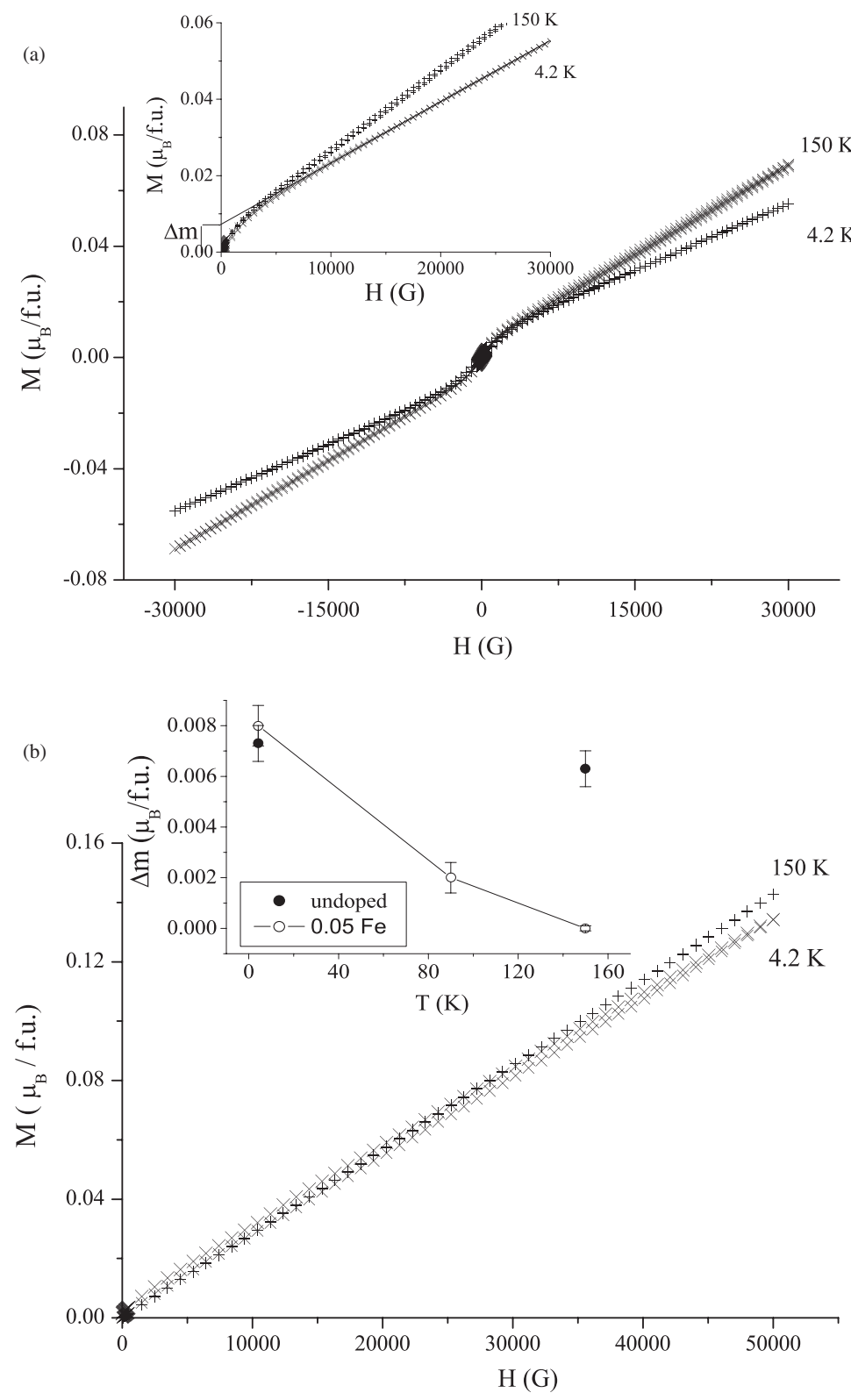

Figure 3. Magnetic field dependence of magnetization: (a) for the undoped $\mathrm{La}_{0.33} \mathrm{Ca}_{0.67} \mathrm{MnO}_{3}$ compound at 4.2 and $150 \mathrm{~K}$. The inset shows the enlarged picture for positive values of the applied magnetic field. Zero-field magnetization $\Delta m$ is defined as a zero-field extrapolation from the highfield slope. (b) For doped $\mathrm{La}_{0.33} \mathrm{Ca}_{0.67} \mathrm{Mn}_{0.95} \mathrm{Fe}_{0.05} \mathrm{O}_{3}$ compound at 4.2 and $150 \mathrm{~K}$. The inset shows the temperature dependence of $\Delta m$ extrapolated from the high-field slope in $M(H)$ for undoped and doped compositions. The line is a guide for eyes.

(Bohr magneton per formula unit). At intermediate temperatures $(150 \mathrm{~K})$, the undoped sample presents approximately the same $\Delta m$ value as at $4.2 \mathrm{~K}$. The recorded remanence contribution $\Delta m<0.01 \mu_{\mathrm{B}} /$ f.u. is less than $0.3 \%$ of the idealized saturated magnetic moment. This 
suggests that the ferromagnetic clusters involve only a small fraction of the Mn spins and hence are short range.

The Fe-doped composition exhibits pure antiferromagnetic behaviour at $150 \mathrm{~K}$ without any presence of ferromagnetic component (see the inset in figure 3(b)). Hence Fe doping suppresses ferromagnetic and favours antiferromagnetic behaviour at intermediate temperatures below $240 \mathrm{~K}$. This is in agreement with the fact that $\mathrm{Fe}^{3+}$ does not participate in double exchange interaction and hence should suppress the ferromagnetic phase. However, at $T=4.2 \mathrm{~K}$, the effect of $\mathrm{Fe}$ on $\Delta m$ becomes negligible. At low temperature, some spin glass or cluster glass contributions could occur [7] and this would need additional investigations.

We carried out the measurements of $M(T)$ well above $T_{\text {co }}$, up to $400 \mathrm{~K}$ (figure 1 ). This allows us to investigate a sufficiently wide temperature interval to discuss the magnetic behaviour above $T_{\text {co }}$ in detail. In the temperature range where short-range ferromagnetic fluctuations are negligible, the susceptibility is expected to follow the Curie-Weiss law:

$$
\chi=\frac{M}{H}=\frac{C^{*}}{T-\theta},
$$

where $C^{*}=\frac{p_{\text {eff }}^{2} \mu_{\mathrm{B}}^{2} N}{3 k_{\mathrm{B}}}, p_{\text {eff }}$ is the effective Bohr magneton number per magnetic ions, $\theta$ is the Weiss temperature and $k_{\mathrm{B}}$ is the Boltzmann constant. Figure 4 shows that the plot of $\chi^{-1}(T)$ versus $T$ can be fitted with the Curie-Weiss law above $320 \mathrm{~K}$. The estimated values of $\theta$ were plotted versus Fe concentration $y$ as shown in figure 2. Similar to $T_{\mathrm{co}}, \theta$ also linearly decreases with $y$ value as

$$
\theta(y)=\theta(y=0)-B^{*} y
$$

for $0 \leqslant y \leqslant 0.06$, where $B^{*}=840 \pm 20 \mathrm{~K}, \theta(y=0) \approx 175 \mathrm{~K}$. It should be noted that both curves in figure 2 are almost parallel to each other.

Using equation (2) we estimated $p_{\text {eff }}$ from experimental curves in figure 4 (a) in the range where they fit well the Curie-Weiss behaviour. The obtained values are shown in figure 4(b) in comparison with the theoretical values of $p_{\text {eff }}^{\text {th }}$ expected for a mixture of $\mathrm{Mn}^{3+}, \mathrm{Mn}^{4+}$ and $\mathrm{Fe}^{3+}$ in ratio $(0.33-y): 0.67: y$, where the average magnetic ion spin is taken as a weighted mean value between those of $\mathrm{Mn}^{3+}(S=2), \mathrm{Mn}^{4+}(S=3 / 2)$ and $\mathrm{Fe}^{3+}(S=5 / 2)$. As seen, the experimentally obtained value of $p_{\text {eff }}$ is $10-13 \%$ higher than the theoretical $p_{\text {eff }}^{\text {th }}$ for all studied compositions. Fe doping does not lead to an increase in $p_{\text {eff }}$, as can be expected from the spin mixture rule, but rather slightly decreases $p_{\text {eff }}$ value.

This enhancement in $p_{\text {eff }}$ value for undoped and Fe doped compositions compared with the calculated value of $p_{\mathrm{eff}}^{\text {th }}$ is not due to an increase of the $g$ factor. Indeed, an EPR measurement at $9.5 \mathrm{GHz}$ carried out with the same sample $(y=0)$ shows at $295 \mathrm{~K}$ a strong EPR resonance (Dysonian line shape with a peak to peak line width $=735 \mathrm{G}$ ) centred at $g=1.996 \pm 0.015$. The enhancement in $p_{\text {eff }}$ can be attributed to the ferromagnetic exchange between the Mn lattice and the conduction electrons with the same $g=2$ as the Mn spins (magnetic polaron).

Now we will estimate the effect of Fe doping on the Weiss temperature $\theta$ in the mean field approximation. Since our EDX analysis confirms a quite uniform distribution of $\mathrm{Fe}^{3+}$ in the parent lattice LCMO and the obtained values of $p_{\text {eff }}$ do not differ much from $p_{\text {eff }}^{\text {th }}$ we can apply this method to the system of $\mathrm{Mn}^{3+}, \mathrm{Mn}^{4+}$ and $\mathrm{Fe}^{3+}$ ions in the range of its paramagnetic behaviour, where it follows the Curie-Weiss law. Let $\mathrm{Mn}^{3+} \equiv A_{1}, \mathrm{Mn}^{4+} \equiv A_{2}$ and $\mathrm{Fe}^{3+} \equiv C$, then for magnetization of $A_{1}, A_{2}$ and $C$ ion subsystems the following system of equations can be written [29]:

$$
\begin{aligned}
& M_{A_{1}}=\chi_{A_{1}}\left(H+\lambda M_{A_{2}}+\varphi M_{A_{1}}+v M_{C}\right), \\
& M_{A_{2}}=\chi_{A_{2}}\left(H+\lambda M_{A_{1}}+\psi M_{A_{2}}+\eta M_{C}\right), \\
& M_{C}=\chi_{C}\left(H+v M_{A_{1}}+\eta M_{A_{2}}\right),
\end{aligned}
$$



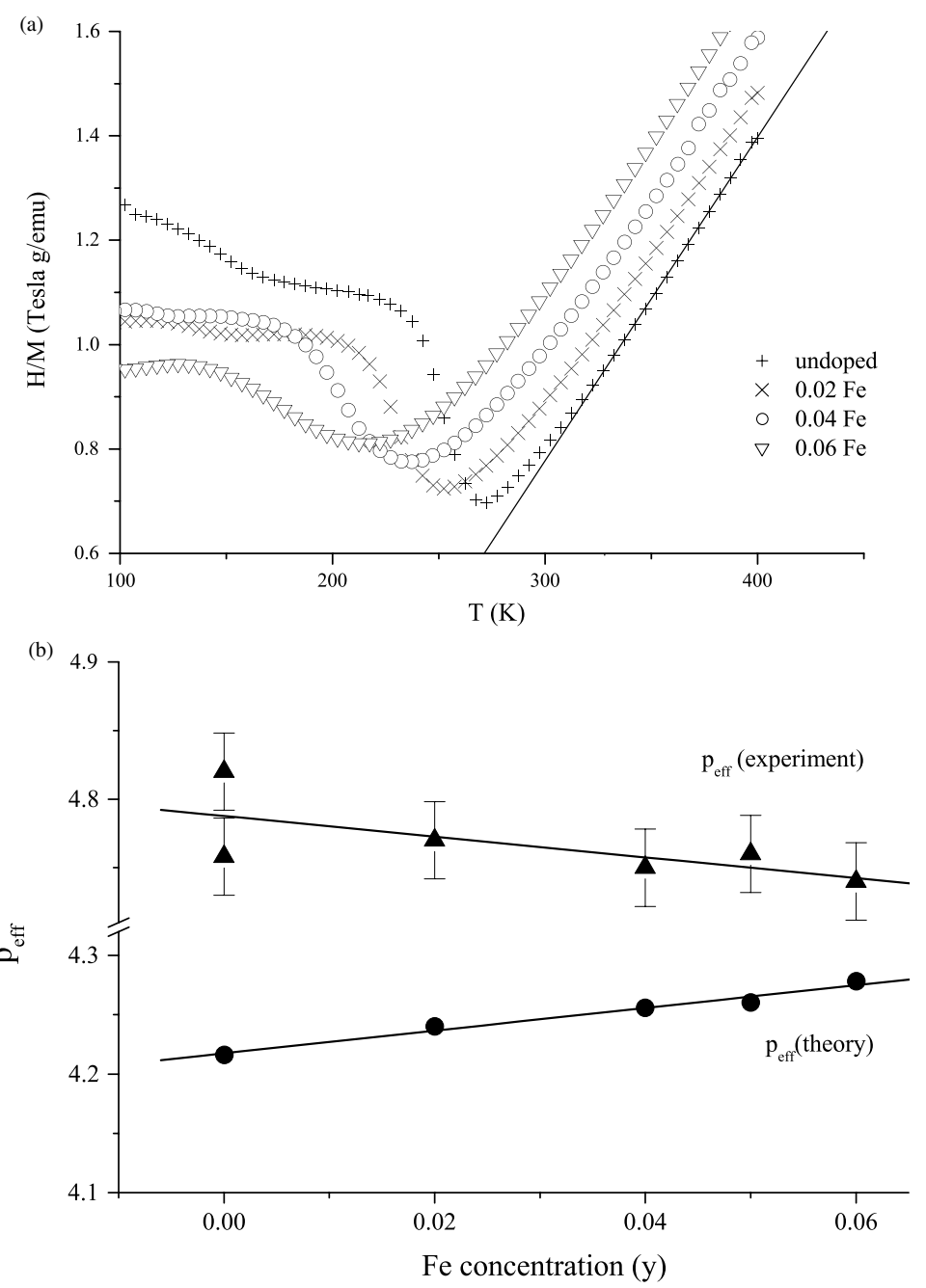

Figure 4. (a) Temperature dependence of the inverse susceptibility $H / M$ of the studied samples at $H=1 \mathrm{~T}$. The solid line displays a fitting of the data to Curie-Weiss behaviour. (b) Dependence of $p_{\text {eff }}$ estimated from the $H / M$ curves in comparison with $p_{\mathrm{eff}}^{\text {th }}$ calculated with the independent spin mixture rule.

where $M_{A_{1}}, M_{A_{2}}$ and $M_{C}$ are magnetizations and $\chi_{A_{1}}, \chi_{A_{2}}$ and $\chi_{C}$ are magnetic susceptibilities of the subsystems of $A_{1}, A_{2}$ and $C$ ions respectively. $\lambda, \varphi, \psi, v$ and $\eta$ are the molecular mean field constants describing the exchange between the three types of magnetic ions. The magnetization we measure is

$$
M=M_{A_{1}}+M_{A_{2}}+M_{C} .
$$

In the limit $T \rightarrow \infty$ we will calculate magnetization in the form of the Curie-Weiss law. To simplify the calculations the following approximations were made for $T>T_{\mathrm{co}}$ :

$$
\begin{aligned}
& \chi_{A_{1}}=\chi_{A_{2}} \equiv \chi^{*}, \\
& \mu_{A_{1}}^{2}=\mu_{A_{2}}^{2}=\mu_{C}^{2} \equiv \mu^{2}, \\
& \psi=\varphi,
\end{aligned}
$$




$$
\eta=v
$$

where $\mu_{A_{1}}, \mu_{A_{2}}$ and $\mu_{C}$ are magnetic moments of $A_{1}, A_{2}$ and $C$ ions, respectively. The above approximations (8)-(11) reflect the fact that the valencies of the Mn atoms are indistinguishable above $T_{\text {co }}$ and their average moment does not differ too much from that of Fe.

Calculating equations (4)-(7) we find the magnetization of the whole system of $A_{1}, A_{2}$ and $C$ ions is given as

$$
\frac{M}{H}=\frac{2 \chi^{*}+\chi_{C}+\chi^{*} \chi_{C}\{4 v-(\lambda+\varphi)\}}{1-(\lambda+\varphi) \chi^{*}-2 v^{2} \chi^{*} \chi_{C}}
$$

For the case of small doping when $\chi_{C}<\chi^{*}$ we approximate $\chi^{*} \chi_{C} \rightarrow 0$, then $M / H$ is given to first order in $\chi_{C}$ as

$$
\frac{M}{H} \approx \frac{2 \chi^{*}+\chi_{C}}{1-(\lambda+\varphi) \chi^{*}-\frac{1}{2} \chi_{C}(4 v-\lambda-\varphi)+0\left(\chi^{*} \chi_{C}\right)} .
$$

In the range of paramagnetic behaviour [29]

$$
\chi=\frac{\mu^{2} N}{3 k_{\mathrm{B}} T},
$$

where $N$ is the number of magnetic particles in the considered system. Then in our system with the number $N$ of $A_{1}, A_{2}$ and $C$ ions and the number $N_{C}$ of $C$ atoms, taking into consideration expressions (8) and (9), $\chi^{*}$ and $\chi_{C}$ are given as

$$
\begin{aligned}
& \chi^{*}=\frac{1}{2} \cdot \frac{\mu^{2}\left(N-N_{C}\right)}{3 k_{\mathrm{B}} T}, \\
& \chi_{C}=\frac{\mu^{2} N_{C}}{3 k_{\mathrm{B}} T}
\end{aligned}
$$

so that

$$
2 \chi^{*}+\chi_{C}=\frac{\mu^{2} N}{3 k_{\mathrm{B}} T} .
$$

With the susceptibilities given in (15)-(17), equation (13) becomes

$$
\frac{M}{H} \approx \frac{N \mu^{2}}{3 k_{\mathrm{B}}\left\{T-\frac{\lambda+\varphi}{2}\left(N-N_{C}\right) \frac{\mu^{2}}{3 k_{\mathrm{B}}}-\frac{N_{C} \mu^{2}}{3 k_{\mathrm{B}}}\left(2 v-\frac{\lambda+\varphi}{2}\right)\right\}} .
$$

Comparing (18) with the expression for the Curie-Weiss law (equation (2)), we obtain the Weiss temperature $\theta$ as

$$
\theta=\frac{(\lambda+\varphi) \mu^{2} N}{6 k_{\mathrm{B}}}+\frac{\mu^{2} N_{C}}{3 k_{\mathrm{B}}}(2 v-\lambda-\varphi) .
$$

Each of the two terms of (19) has a simple physical meaning. Indeed if $v=0$ then

$$
\theta=\frac{\mu^{2}}{3 k_{\mathrm{B}}}(\lambda+\varphi)\left(\frac{N}{2}-N_{C}\right)
$$

describing the decrease of the Weiss temperature with the doping by non-magnetic impurities (such as $\mathrm{Ru}$, for example), whereas if $\lambda=\varphi=0$ then

$$
\theta=\frac{2 \mu^{2} v}{3 k_{\mathrm{B}}} N_{C}
$$

showing the increase of the Weiss temperature with the doping by magnetic impurities in the presence of the non-interacting parent background.

The Weiss temperature $\theta(y)$ (equation (19)) as a function of concentration of impurity $\mathrm{Fe}^{3+}, y=N_{C} / N$, is given as

$$
\theta(y)=\theta(0)\left\{1-2 y\left(1-\frac{2 \nu}{\lambda+\varphi}\right)\right\},
$$


where

$$
\theta(0)=\frac{(\lambda+\varphi) \mu^{2} N}{6 k_{\mathrm{B}}}
$$

is the Weiss temperature in the undoped sample, i.e. when $y=0$.

Comparing (22) with (3) we get an expression for the slope $B^{*}$ :

$$
B^{*}=2 \theta(0)\left(1-\frac{2 v}{\lambda+\varphi}\right) \text {. }
$$

Taking $B^{*}=(840 \pm 20) \mathrm{K}$ and $\theta(y=0) \approx 175 \mathrm{~K}$ yields the ratio of the (Fe-Mn) to $(\mathrm{Mn}-\mathrm{Mn})$ molecular fields:

$$
\frac{2 v}{\lambda+\varphi}=-1.40 \pm 0.06
$$

The negative sign shows that the exchange coupling between the Fe and Mn ions is opposite to that between the $\mathrm{Mn}$ ions. In order to evaluate the order of magnitude of the averaged Heisenberg exchange $J(\mathrm{Mn}-\mathrm{Mn})$, similarly to the work of Causa et al [30] we adapt the mean field relation derived for a ferromagnetic lattice to LCMO [29]:

$$
\begin{aligned}
& J(\mathrm{Mn}-\mathrm{Mn})=\frac{g^{2} \mu_{\mathrm{B}}^{2} N}{2 z}(\lambda+\varphi), \\
& J(\mathrm{Mn}-\mathrm{Fe})=\frac{g^{2} \mu_{\mathrm{B}}^{2} N v}{z},
\end{aligned}
$$

where $g$ is the gyromagnetic ratio, $z$ is the number of first neighbour $\mathrm{Mn}$ in the perovskite structure and $N$ is the number of Mn per unit volume. Using (23) and taking $z=6$ and $g=2$, we get

$$
J(\mathrm{Mn}-\mathrm{Mn})=\frac{2 k_{\mathrm{B}} \theta(0)}{p_{\mathrm{eff}}^{2}} .
$$

Taking $p_{\text {eff }}=4.75 \mu_{\mathrm{B}}$ and $\theta(0)=175 \mathrm{~K}$ from figure 4 gives $J(\mathrm{Mn}-\mathrm{Mn}) / k_{\mathrm{B}}=15.5 \mathrm{~K}$ and, since

$$
\frac{2 v}{\lambda+\varphi}=\frac{J(\mathrm{Fe}-\mathrm{Mn})}{J(\mathrm{Mn}-\mathrm{Mn})}
$$

we get $J(\mathrm{Fe}-\mathrm{Mn}) / k_{\mathrm{B}}=-22 \mathrm{~K}$. The minus sign shows an antiferromagnetic coupling for $J(\mathrm{Fe}-\mathrm{Mn})$ as expected a priori from the fast decrease of the Weiss temperature with $\mathrm{Fe}$ doping.

It is important to note that all samples showed positive $\theta$ values, which means that, in our case, ferromagnetic interactions are dominant for $T>T_{\mathrm{co}}$. On the other hand, all studied samples exhibit predominant antiferromagnetic insulating ground state at low temperature. This means that in the studied compounds the magnetic ordering is determined by charge ordering.

\subsection{Electrical transport properties}

The electric resistivity $\rho$ as a function of temperature was measured for all studied compositions. Figure 5(a) displays such dependences for most of them. The other compositions showed a similar behaviour and are omitted here so as not to overload the figure. The character of the $\rho(T)$ dependences is kept after Fe doping and the value of resistivity does not change substantially in the whole doping range $0 \leqslant x \leqslant 0.06$. The samples remain essentially semiconducting over the entire temperature range. As seen in figure 5(a) the resistivity data could not be fitted to a single exponential temperature dependence. In the 

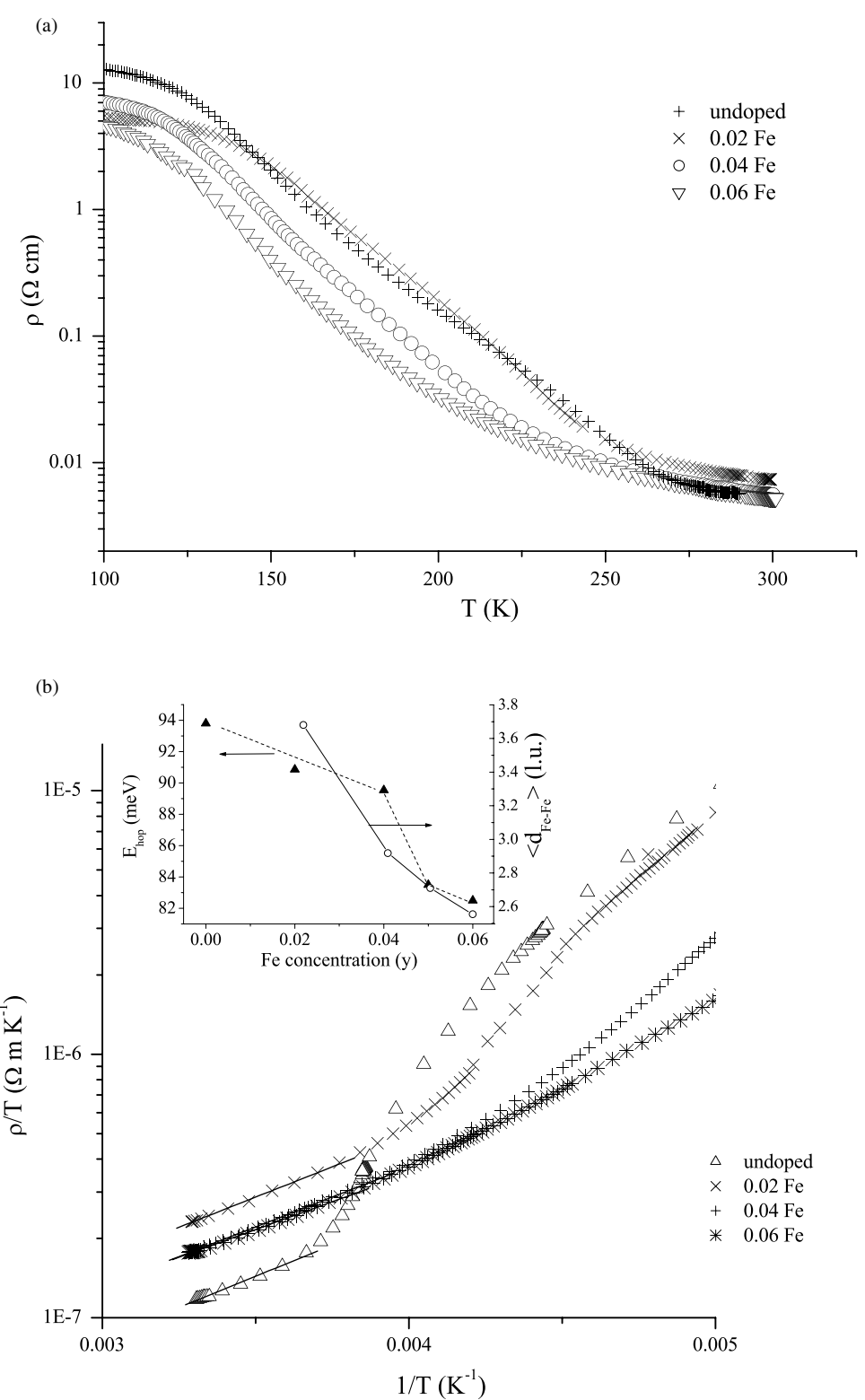

Figure 5. (a) Temperature dependence of resistivity of $\mathrm{La}_{0.33} \mathrm{Ca}_{0.67} \mathrm{Mn}_{1-y} \mathrm{Fe}_{y} \mathrm{O}_{3}$ with different Fe concentrations $y$. (b) Plots of $\lg (\rho / T)$ versus $1 / T$. The solid lines correspond to polaronic conduction. Inset: average Fe-Fe separation and the activation energy $E_{\text {hop }}$ upon Fe concentration.

temperature range of paramagnetic behaviour, the small polaron hopping mechanism described by $\rho(T)=A T \exp \left(E_{\mathrm{hop}} / T\right)$ and used to explain the resistivity for other manganites by Jaime et al [31] seems to provide the best fitting to the data (figure 5(b)). The inset in figure 5(b) displays the dependence of the estimated values of $E_{\text {hop }}$ with doping level $y, E_{\text {hop }}$ being obtained from $\lg (\rho / T)$ versus $1 / T$ plots. In the same figure the average $\mathrm{Fe}-\mathrm{Fe}$ separation in terms of lattice units $(1$ l.u. $=3.87 \AA)$ is also shown. 
The obtained values of $E_{\mathrm{hop}}$ are very close to each other and comparable to the value $\sim 100 \mathrm{meV}$ found in [19] for $\mathrm{La}_{0.3} \mathrm{Ca}_{0.7} \mathrm{Mn}_{0.8} \mathrm{Cr}_{0.2} \mathrm{O}_{3}$ and to the value of $100-110 \mathrm{meV}$ for $\mathrm{La}_{0.75} \mathrm{Ca}_{0.25} \mathrm{Mn}_{1-y} \mathrm{Fe}_{y} \mathrm{O}_{3}(0 \leqslant y \leqslant 0.05)$ [20], but almost twice as high as the value of $45 \mathrm{meV}$ found for $\mathrm{La}_{0.35} \mathrm{Ca}_{0.65} \mathrm{MnO}_{3}$ above its $\mathrm{CO}$ transition [27]. As seen in the inset in figure 5(b), $E_{\text {hop }}$ slightly decreases with $y$-value. Fe does not participate in double exchange; consequently, the carrier hopping occurs between the $\mathrm{Mn}^{3+}$ and $\mathrm{Mn}^{4+}$ ions even in Fe-doped samples [20], and hence the activation energy is not expected to change significantly, as is observed. However, Fe doping leads to a decrease in $\mathrm{Mn}^{3+}$ concentration, which could slightly increase $E_{\text {hop. }}$. On the contrary, a slight decrease of $E_{\text {hop }}$ was found in Fe-doped samples.

Nevertheless, at higher Fe concentrations, which have not been considered in this paper, $E_{\text {hop }}$ is most likely to increase and the resistivity of samples will be enhanced. Ahn et al [21] analysed the influence of a high level of Fe doping (9-20\% Fe) on the resistivity of $\mathrm{La}_{0.47} \mathrm{Ca}_{0.53} \mathrm{Mn}_{1-y} \mathrm{Fe}_{y} \mathrm{O}_{3}$. A pronounced increase of the resistivity was found in the paramagnetic range at room temperature, for $y>0.13$, whereas the resistivity of sample with $y=0.09$ was slightly lower than the resistivity of the undoped composition. This is in good agreement with our findings.

\subsection{Electron microscopy experiments}

The influence of Fe doping on charge ordering structure was directly investigated by transmission electron diffraction and microscopy (FEG-TEM) on doped $\mathrm{La}_{1 / 3} \mathrm{Ca}_{2 / 3} \mathrm{Mn}_{0.95} \mathrm{Fe}_{0.05} \mathrm{O}_{3}$ manganites in comparison with undoped $\mathrm{La}_{0.33} \mathrm{Ca}_{0.67} \mathrm{MnO}_{3}$. Electron diffraction patterns and high resolution lattice images were achieved mostly with a [001] zone-axis in the temperature range extending from 90 to $300 \mathrm{~K}$.

In both the undoped (parent) compound and the $5 \% \mathrm{Fe}$ doped one, electron diffraction (ED) patterns exhibit identical Pbnm-type structure at room temperature (figure 6(a)), whereas at low $T(91 \mathrm{~K})$ charge ordering gives rise to extra spots at positions between the main Bragg peaks. Such a characterization of the charge ordering in $\mathrm{La}_{0.33} \mathrm{Ca}_{0.67} \mathrm{MnO}_{3}$ at $91 \mathrm{~K}$ is shown in figure 6(b), which displays a [001] zone-axis electron diffraction pattern obtained from an area selected by an aperture introduced in the Gauss plane. The sharp weaker satellite spots that can be indexed traditionally as $\boldsymbol{q}=(1 / 3-\varepsilon) \boldsymbol{a}^{*}$ ( $\varepsilon$ is the parameter of incommensurability, in figure $6(\mathrm{~b}) \varepsilon=0$ ) are superlattice spots due to charge ordering. The formation of such superstructure in the parent composition $\mathrm{La}_{0.33} \mathrm{Ca}_{0.67} \mathrm{MnO}_{3}$ is known and reported, for example, in $[13,14]$. It was found that at temperature $91-92 \mathrm{~K}$ the $\mathrm{CO}$ seems to be typical for the whole sample.

The undoped sample displayed charge ordering with $\boldsymbol{q}$-vector equal to $1 / 3 \boldsymbol{a}^{*}$ (figure $6(\mathrm{c})$ ). The difference in the measured values did not exceed $5 \%$, that is within the error in the measurement. Thus, it could be concluded that in the undoped sample, at low temperature $T=91-92 \mathrm{~K}$, the CO has a commensurate character. On the other hand, in the doped sample, at low temperature $91-92 \mathrm{~K}$ the $\boldsymbol{q}$-value varies from 0.28 to 0.31 within the same grain and from one grain to another: the average $\boldsymbol{q}$-value is about $12-15 \%$ smaller than in the undoped sample as it is seen from the comparison of $q$-values for undoped and doped samples in figure $6(\mathrm{c})$. Consequently, it can be inferred that charge ordering has an incommensurate character in the Fe doped manganite.

The significant parameters for $\mathrm{CO}$ were assessed by Van Tendeloo et al [15] to be the temperature, the $\mathrm{La} / \mathrm{Ca}$ ratio, which determines the $\mathrm{Mn}^{3+} / \mathrm{Mn}^{4+}$ ratio, and the oxygen content for a given $x$, which also affects the $\mathrm{Mn}^{3+} / \mathrm{Mn}^{4+}$ ratio. According to Chen and Cheong [13], the $q$-value is given by $q \sim(1-x)$ for manganites corresponding to the formula of $\mathrm{La}_{1-x} \mathrm{Ca}_{x} \mathrm{MnO}_{3}$. 


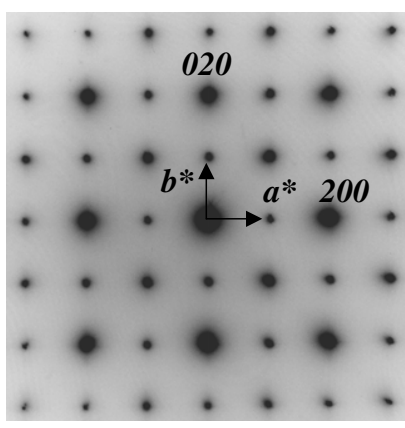

(a)

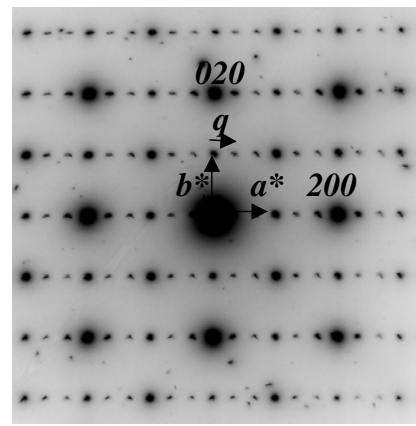

(b)

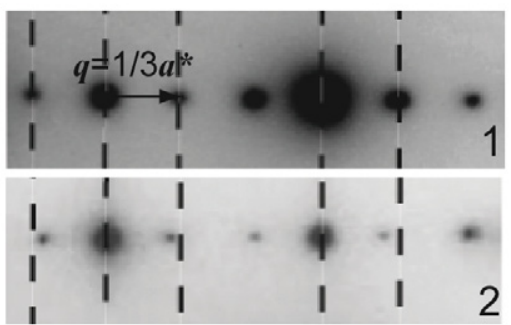

(c)

Figure 6. [001] Electron diffraction patterns of $\mathrm{La}_{0.33} \mathrm{Ca}_{0.67} \mathrm{MnO}_{3}$ recorded at room temperature (a) and at $91 \mathrm{~K}(\mathrm{~b})$. The satellite reflections are superlattice spots due to charge ordering $\left(\boldsymbol{q}=1 / 3 \boldsymbol{a}^{*}\right)$. (c) Comparison of diffraction patterns recorded at $91 \mathrm{~K}$ for undoped $\mathrm{La}_{0.33} \mathrm{Ca}_{0.67} \mathrm{MnO}_{3}$ (1: enlarged (b)) and doped $\mathrm{La}_{0.33} \mathrm{Ca}_{0.67} \mathrm{Mn}_{0.95} \mathrm{Fe}_{0.05} \mathrm{O}_{3}$ (2) samples.

In both studied compounds, doped and undoped, our EDX analysis confirms the same value of the $\mathrm{La} / \mathrm{Ca}$ ratio as shown above. Iodometric titration gives similar values for the oxygenation level (table 2). Hence, the only factor which causes a change in $\mathrm{Mn}^{3+} / \mathrm{Mn}^{4+}$ ratio seems to be the substitution of $\mathrm{Mn}^{3+}$ by $\mathrm{Fe}^{3+}$. In the undoped composition $\mathrm{Mn}^{3+}: \mathrm{Mn}^{4+}=1: 2$, whereas in the $5 \%$ Fe doped one $\mathrm{Mn}^{3+}: \mathrm{Mn}^{4+}=0.85: 2$, which brings about a $15 \%$ decrease in the $\mathrm{Mn}^{3+} / \mathrm{Mn}^{4+}$ ratio. This is in good quantitative agreement with the obtained difference in $q$-value for the doped and undoped compounds. Thus the difference in $q$-value is compatible with the change in $\mathrm{Mn}^{3+} / \mathrm{Mn}^{4+}$ ratio.

The electron diffraction patterns were recorded versus temperature under the same optical and geometrical experimental conditions. The temperature was first lowered down to $90 \mathrm{~K}$ and then increased from 90 to $300 \mathrm{~K}$, by steps of $10^{\circ}-20^{\circ}$. Figure 7 displays the evolution of the $q$-value versus $T$ for the undoped and $5 \%$ Fe-doped compounds. The $q$-value of the parent composition is equal to $1 / 3$ at $91 \mathrm{~K}$ and remains unchanged between $91 \mathrm{~K}$ and $T_{\text {plat }} \sim 170$ $200 \mathrm{~K}$. For the fully oxygenated composition with $x=0.5$ similar behaviour with $T_{\text {plat }}=160 \mathrm{~K}$ was observed in [16]. The authors associated this $T_{\text {plat }}$ with the Néel temperature, $T_{\mathrm{N}}$, of CEtype antiferromagnetic structure [11]. However, in our case (figure 7), we did not observe such a sharp decrease of $q$-value at a precise $T_{\text {plat }}$ and can only note a tendency to start a slight decrease of $q$-value from 170-200 K. This slight decrease goes on down to $260 \mathrm{~K}$, where a drastic change of the intensity and shape of the $\mathrm{CO}$ extra spots occurs in the narrow temperature interval of $260-270 \mathrm{~K}$. The satellite reflections give rise to streaking and then disappear completely at $T \approx 270 \mathrm{~K}$. Thus, the observed structural transition in the undoped sample is in a good agreement with the $T_{\text {co }}$ found in $M(T)$ measurements. 


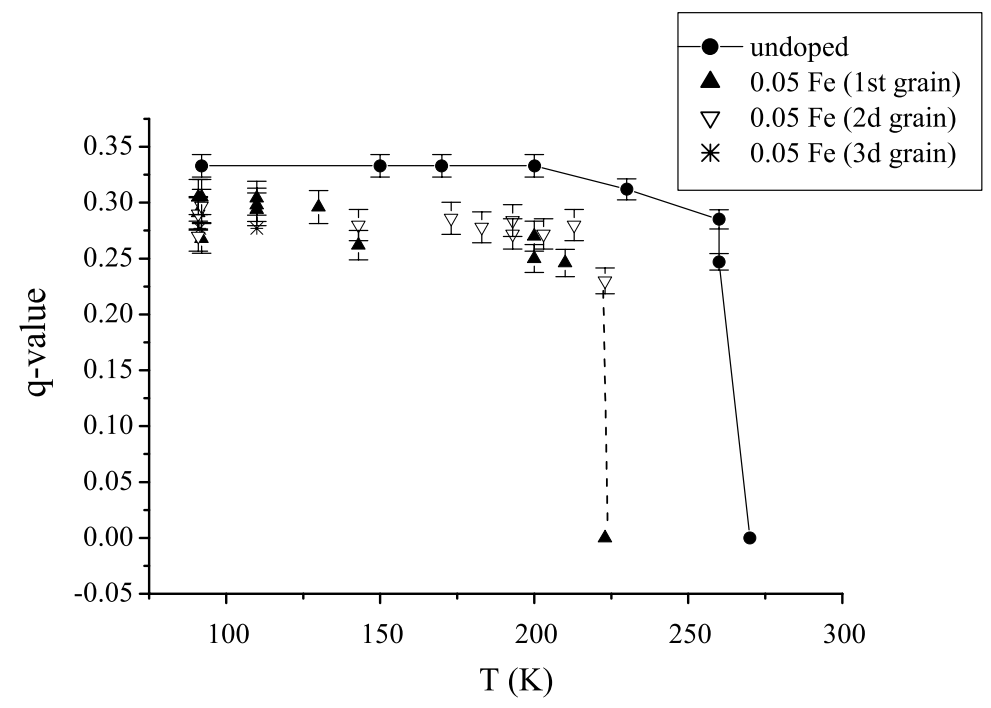

Figure 7. $q$-value as a function of temperature for undoped $\mathrm{La}_{0.33} \mathrm{Ca}_{0.67} \mathrm{MnO}_{3}$ and doped $\mathrm{La}_{0.33} \mathrm{Ca}_{0.67} \mathrm{Mn}_{0.95} \mathrm{Fe}_{0.05} \mathrm{O}_{3}$ compositions.

In the Fe doped sample (figure 7), the $q$-value is stable when $T$ increases from 90 to $200 \mathrm{~K}$. Towards $220 \mathrm{~K}$ satellite spots became diffused along the $\boldsymbol{a}^{*}$ direction and sharp spots disappear completely at $T \sim 220 \mathrm{~K}$. This phenomenon occurs at about $50^{\circ}$ under $T_{\text {co }}$ found for undoped samples. Such a behaviour for $q(T)$ also fits $M(T)$ measurements. However, in the case of the doped compound the diffraction patterns still exhibit a streaking between Bragg spots along the $\boldsymbol{a}^{*}$ direction up to $240-250 \mathrm{~K}$, i.e. at temperatures much higher than $T_{\mathrm{co}}$. It must be pointed out that such a streaking was not found everywhere but only in limited areas within a grain. Figure 8 presents an example of evolution of diffraction patterns with temperature for the doped composition. A weak streaking can be observed around Bragg spots at $T>T_{\text {co. }}$. Such streaking could indicate that in the Fe-doped sample partial charge ordering could remain (to a lesser extent and in small local areas) at temperatures higher than $T_{\mathrm{co}}$. These structural features cannot be seen from $M(T)$ dependence.

To better understand the influence of Fe doping on the charge ordering process and the corresponding structure of the incommensurability in the doped sample, at low and room temperature, high resolution electron microscopy (HREM) images were recorded for undoped and $5 \%$ Fe-doped compounds. No special features were found in the Fe-doped composition compared with undoped at room temperature.

Figure 9(a) shows a typical [001] structure image of the undoped sample at $91 \mathrm{~K}$. To enhance the phase contrast, similar to [32], the image was processed by Fourier filtering of the FFT (figure 9(b)) and reconstructed in the inverse FFT (figure 9(c)) with a special mask where all visible reflections (including main and satellite reflections) were selected. The size and arrangement of applied masks are shown in figure 9(d). From figures 9(a) and (c), the periodicity of the superstructure can be visualized and measured precisely by using the substrate as a template. A spacing close to $16.5 \AA$ was found for this superlattice. It corresponds to a threefold increase of the parameter $\left(3 a_{p} \sqrt{2}\right)$ along the $a$ direction, which is in agreement with the value: $q=1 / 3 a^{*}$ in reciprocal space. This fits the expected 1:2 ordering of JahnTeller heavy distorted $\mathrm{Mn}^{3+} \mathrm{O}^{6}$ stripes and non-distorted $\mathrm{Mn}^{4+} \mathrm{O}^{6}$ stripes as previously reported 

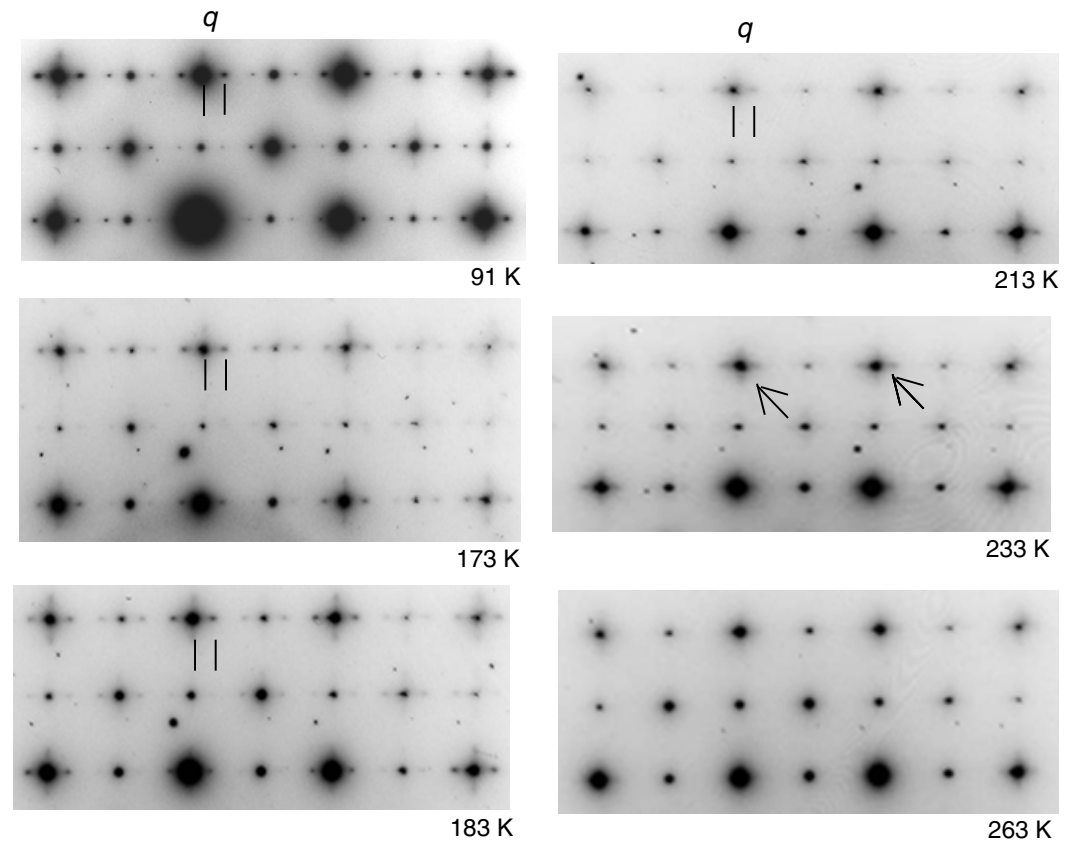

Figure 8. Evolution of satellite reflection with $\boldsymbol{q}=(1 / 3-\varepsilon) \boldsymbol{a}^{*}$ with increasing temperature in $\mathrm{La}_{0.33} \mathrm{Ca}_{0.67} \mathrm{Mn}_{0.95} \mathrm{Fe}_{0.05} \mathrm{O}_{3}$. Arrows show remaining streaking near Bragg reflections.

in $[13,14]$. This regular single or twinned superstructure is found at low temperature all over the grains.

The [001] HREM image obtained at $91 \mathrm{~K}$ for the $5 \%$ Fe doped sample is shown in figure 10(a) with the corresponding FTT (figure 10(b)) and inverse FTT (figure 10(c)) carried out as in figure 9 . This crystal with $q=(1 / 3-\varepsilon) a^{*}(\varepsilon \approx 0.05)$ value also displays an extended superstructure. However, it was found that the superstructure was not as regular as in the undoped sample. In order to get more detail on the distribution of this structure all along the grains, we carried out dark field images obtained by interference of one diffracted spot with an adjacent satellite spot as shown in the diffraction pattern in the figure 11. The direct interpretation of such an image is difficult, but its comparison with the same type of dark field image obtained for the undoped sample, which is perfectly regular, is striking. An estimation of the periodicity of the superstructure yields 18-20 $\AA$, that fits well with the obtained decrease in $q$-value of about $15 \%$ for this sample. It can also be inferred that the superstructure is disturbed locally all over the crystals and faults occur in the arrangements of stripes (figure 11). Shifts up to one lattice parameter $\left(=a_{p} \sqrt{2}\right)$ are observed in figure 11. Shifts corresponding to $a_{p} \sqrt{2}$ were found in some other manganite systems, for example, in $\mathrm{Nd}_{0.5} \mathrm{Ca}_{0.5} \mathrm{Mn}_{1-y} \mathrm{Cr}_{y} \mathrm{O}_{3}$ [16]. These shifts can give rise to local change in periodicity of $\mathrm{Mn}^{3+} \mathrm{O}_{6}$ and $\mathrm{Mn}^{4+} \mathrm{O}_{6}$ stripes. The substitution of $\mathrm{Mn}^{3+}$ by the $\mathrm{Fe}^{3+}$ ion, which does not participate in orbital ordering, seems to lead to a defect in the formation of $\mathrm{Mn}^{3+} \mathrm{O}_{6}$ chains and, as a result, shifts and faults in stripe ordering can occur. Consequently, doping by Fe is likely to generate the so-called impurity effect, inducing a pinning of the discommensurations. The pinning effect of Ru dopant in the $\mathrm{SmCaMnRuO}$ manganite system was noted in [15]. The occurrence of such defects driving the incommensurability of the superstructure is likely to be responsible for the decrease in $q$-value found in the Fe-doped compounds. 


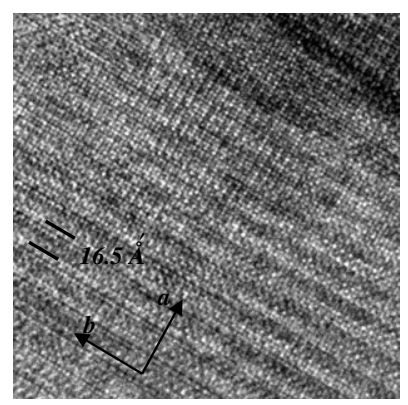

(a)

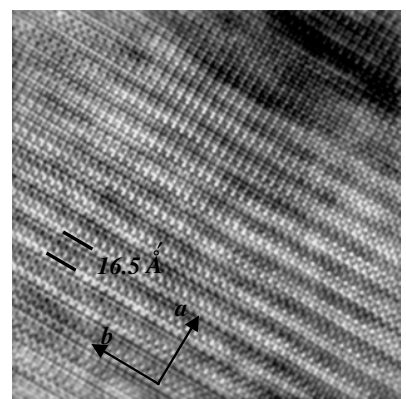

(c)

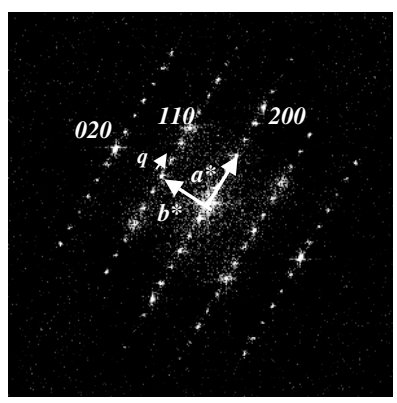

(b)

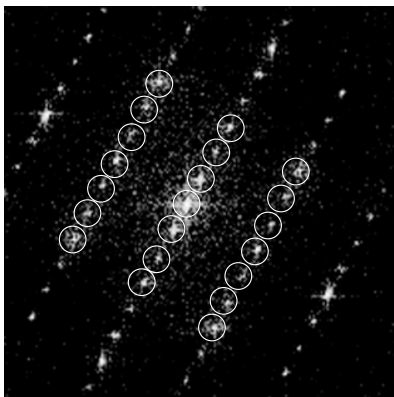

(d)

Figure 9. (a) HREM images of $\mathrm{La}_{0.33} \mathrm{Ca}_{0.67} \mathrm{MnO}_{3}$ composition at $91 \mathrm{~K}$. The periodicity of the superstructure $16.5 \AA=3 a$. (b) FFT of the same area. (c) Processed inverse FFT obtained from (b) with masks selecting all visible reflections as exemplified in (d). (d) Enlarged diffraction pattern of (b) with applied masks.

The obtained linear decrease of charge ordering temperature $T_{\text {co }}$ with the iron impurity concentration $y$ (equation (1)) could be related to the linear decrease of Jahn-Teller mean field with substitution of the $\mathrm{Mn}^{3+} \mathrm{Jahn}-\mathrm{Teller}$ centre (JTC) by non-JTC Fe ${ }^{3+}$. The charge ordering critical temperature $T_{\mathrm{co}}$ is determined as a result of the competition between the mean field induced by $\mathrm{Mn}^{3+} \mathrm{JTCs}, M_{1}=D n_{\mathrm{Mn}^{3+}}$, on the one hand, and the thermal factor, $k_{\mathrm{B}} T$, on the other. Here $n_{\mathrm{Mn}^{3+}}$ is an $\mathrm{Mn}^{3+}$ ion concentration and $D$ is a coefficient proportional to the average energy of indirect vibronic interaction between two $\mathrm{Mn}^{3+} \mathrm{JTCs}$. Namely, the $T_{\text {co }}$ is determined by

$$
k_{\mathrm{B}} T_{\mathrm{co}}^{\mathrm{Mn}^{3+}}=D n_{\mathrm{Mn}^{3+}} .
$$

Substitution of a fraction of $\mathrm{Mn}^{3+}$ JTC by $\mathrm{Fe}^{3+}$ non-JTC with concentration $y$ leads to a monotonic decrease versus $y$ of the Jahn-Teller mean field related now only to the remaining $\mathrm{Mn}^{3+}$ ions. The latter results in a corresponding linear decrease versus $y$ of critical temperature $T_{\text {co }}$ in agreement with (30)

$$
k_{\mathrm{B}} T_{\mathrm{co}}^{\left(\mathrm{Mn}^{3+}-\mathrm{Fe}^{3+}\right)}=D\left(n_{\mathrm{Mn}^{3+}}-y\right) .
$$

Taking $T_{\text {co }}=272 \mathrm{~K}$ obtained for undoped composition $(y=0)$ we get from equation (30) an estimated value of $D \approx 817 \pm 6 \mathrm{~K}$, which should correspond to the measured value: $A^{*}=922 \pm 38 \mathrm{~K}$ (equation (1)). Thus, the experimentally obtained behaviour of $T_{\mathrm{co}}$ as a function of $\mathrm{Fe}$ concentration is in line with the mean field approximation. 


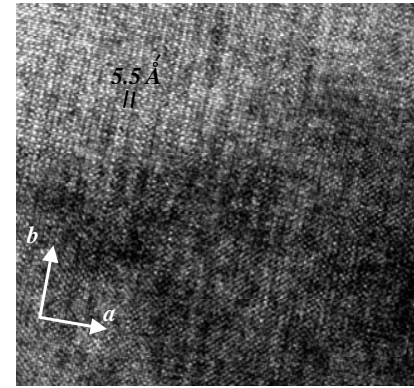

(a)

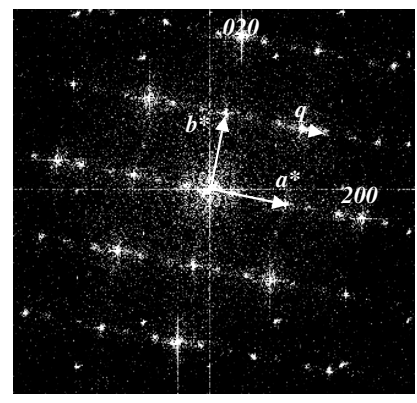

(b)

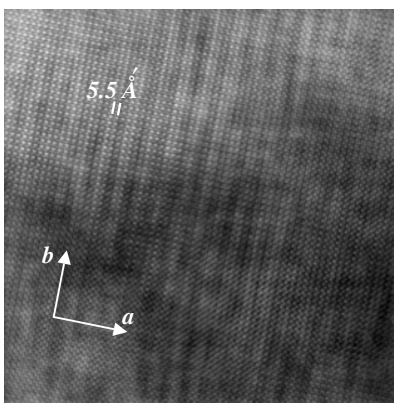

(c)

Figure 10. (a) HREM images of $\mathrm{La}_{0.33} \mathrm{Ca}_{0.67} \mathrm{Mn}_{0.95} \mathrm{Fe}_{0.05} \mathrm{O}_{3}$ at $91 \mathrm{~K}$. (b) FFT of the same area. (c) Processed inverse FFT obtained from (b) with the same conditions as in figure 9.

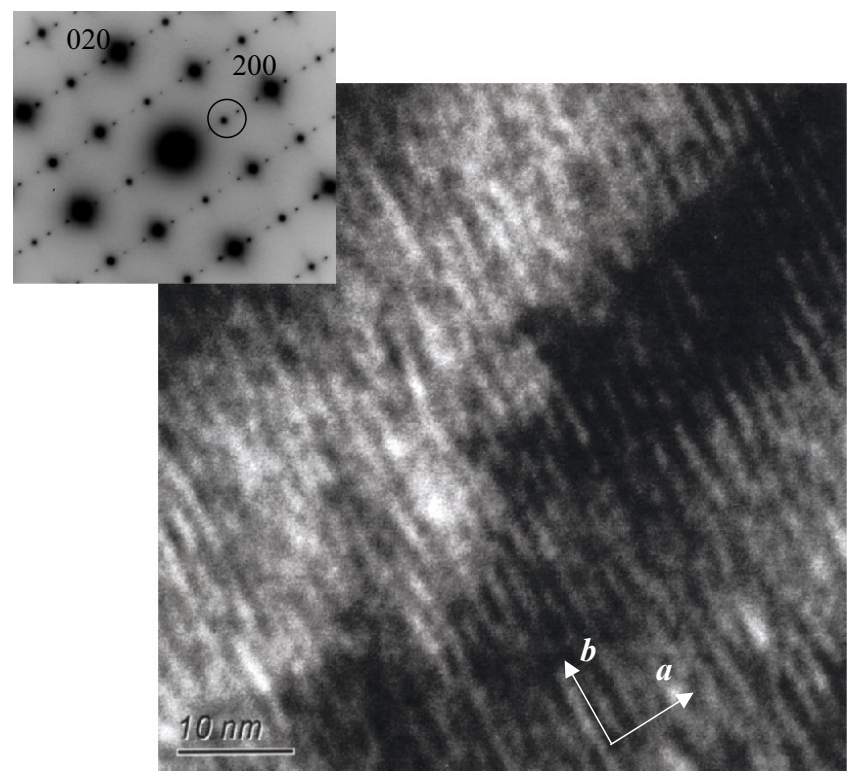

Figure 11. Dark field HREM image of superstructure obtained by interference of one diffracted spot with an adjacent satellite reflection for $\mathrm{La}_{0.33} \mathrm{Ca}_{0.67} \mathrm{Mn}_{0.95} \mathrm{Fe}_{0.05} \mathrm{O}_{3}$. The mode of spot selection is shown in the inset diffraction pattern. 


\section{Conclusion}

The influence of $\mathrm{Fe}$ doping on structure, charge ordering, magnetic and transport properties of $\mathrm{La}_{0.33} \mathrm{Ca}_{0.67} \mathrm{Mn}_{1-y} \mathrm{Fe}_{y} \mathrm{O}_{3}(0 \leqslant y \leqslant 0.06)$ versus temperature has been analysed by collating magnetic and electrical measurements with electron diffraction and high resolution electron microscopy in the range of $90-300 \mathrm{~K}$. The transition at $272 \mathrm{~K}$ attributed to charge ordering in the parent $\mathrm{La}_{0.33} \mathrm{Ca}_{0.67} \mathrm{MnO}_{3}$ composition is suppressed by $\mathrm{Fe}$ doping of $\mathrm{Mn}$ sites. The charge ordering temperature $T_{\text {co }}$ decreases linearly with Fe concentration $y$.

The Weiss temperature $\theta$ estimated from the range of paramagnetic behaviour also decreases linearly with $y$. The observed $\theta$ decrease fits well the mean field approximation. Despite the fact that the obtained values of $\theta$ are positive, all the studied compositions show antiferromagnetic insulating behaviour at low temperature. This is associated with the fact that the charge ordering transition takes place at higher temperature and it seems to determine lower temperature magnetic behaviour. The observed irreversibility at temperatures below $T_{\text {co }}$ is found to be associated with the presence of a small ferromagnetic component, which is suppressed by $\mathrm{Fe}^{3+}$ dopant at intermediate temperatures.

In the undoped samples, electron diffraction versus temperature studies in TEM show predominantly a commensurate superstructure with $q$-value equal to $1 / 3 a^{*}$ over the whole temperature range $<200 \mathrm{~K}$. The Fe-doped composition $\mathrm{La}_{0.33} \mathrm{Ca}_{0.67} \mathrm{Mn}_{0.95} \mathrm{Fe}_{0.05} \mathrm{O}_{3}$ exhibits an incommensurate superstructure at low temperatures, with $q$-value smaller by about $15 \%$, that is comparable with the decrease in $\mathrm{Mn}^{3+} / \mathrm{Mn}^{4+}$ ratio due to substitution of $\mathrm{Mn}^{3+}$ by $\mathrm{Fe}^{3+}$. In both undoped and $\mathrm{Fe}$-doped compositions, the values of charge ordering transition temperature obtained from the $q(T)$ behaviour are in good agreement with $T_{\text {co }}$ values measured from $M(T)$ dependences.

High resolution electron microscopy images provide a better understanding of the charge ordering. The low-temperature superstructure unit of the Fe-doped manganite does not correspond exactly to the threefold cell with lattice parameters $3 a, b, c$; defects including shifts along the $\boldsymbol{a}$ direction induce a disturbance of long-range charge ordering. The charge ordering superstructure formation as well as its suppression by $\mathrm{Fe}^{3+}$ dopants are shown to be related to the Jahn-Teller effect on $\mathrm{Mn}^{3+}$ centres.

\section{Acknowledgments}

The authors acknowledge the support of Conseil Regional de l'Ile de France and Mairie de Paris for our transmission electron microscope (FEG-TEM).

They would also like to thank Sawako Nakamae for friendly help with SQUID magnetometer operation, G Vetter for her help with X-ray powder diffraction measurements, Martin Zak for his help for HREM image processing and A Hohulin for fruitful discussion.

\section{References}

[1] Rao C N R and Raveau B (ed) 1998 Colossal Magnetoresistance, Charge Ordering and Related Properties of Manganese Oxides (Singapore: World Scientific)

[2] Cocy J, Viret M and Molnar S 1999 Adv. Phys. 48167

[3] Salamon M B and Jaime M 2001 Rev. Mod. Phys. 73583

[4] Zener C 1951 Phys. Rev. 82403

[5] Miller A J, Shraiman B I and Mueller R 1996 Phys. Rev. B 545389

[6] Nagaev E L 2002 Colossal Magnetoresistance and Phase Separation in Magnetic Semiconductors (London: Imperial College Press)

[7] Dagotto E, Hotta H and Moreo A 2001 Phys. Rep. 3441 
[8] Goodenouph J P 1955 Phys. Rev. 100564

[9] Schiffer P, Ramirez A P, Bao W and Cheong S-W 1995 Phys. Rev. Lett. 753336

[10] Ramirez A P, Schiffer P, Cheong S-W, Chen C H, Bao W, Palastra T T H, Gammel P L, Bishop D J and Zegarski B 1996 Phys. Rev. Lett. 763188

[11] Wollan E O and Koehler W C 1955 Phys. Rev. 100545

[12] Chen C H and Cheong S-W 1996 Phys. Rev. Lett. 764042

[13] Chen C H, Cheong S-W and Hwang H Y 1997 J. Appl. Phys. 811326

[14] Mori S, Chen C H and Cheong S-W 1998 Nature 392473

[15] Van Tendeloo G, Lebedev O I, Herview M and Raveau B 2004 Rep. Prog. Phys. 671315

[16] Schuddinck W, Van Tendeloo G, Martin C, Hervieu M and Raveau B 2002 J. Alloys Compounds 33313

[17] Barnabe A, Maignan A, Hervieu M, Dainay F, Martin C and Raveau B 1997 Appl. Phys. Lett. 7126

[18] Raveau B, Maignan A, Martin C and Hervieu M J 1997 Solid State Chem. 130162

[19] Sudyoadsuk T, Suryanarayanan R, Winotai P and Wenger L E 2004 J. Magn. Magn. Mater. 27896

[20] Ogale S B, Shreekala R, Bathe R, Date S K, Patil S I, Hannoyer B, Petit F and Marest G 1998 Phys. Rev. B 577841

[21] Ahn K H, Wu X W, Liu K and Chien C L 1996 Phys. Rev. B 5415299

[22] Laiho R, Lisunov K G, Lähderanta E, Salminen J, Shakhov M A, Stamov V S, Petrenko P A and Zakhvalinskii V S 2003 J. Phys. Chem. Solids 641573

[23] Levy P, Granja L, Indelicato E, Vega D, Polla G and Parisi F 2001 J. Magn. Magn. Mater. 226794

[24] Roy M, Mitchell J F, Ramirez A P and Schiffer P 1999 J. Phys.: Condens. Matter 114843

[25] Wu M C, Chen J and Jin X 1997 Physica C 276132

[26] Radeaelli P G, Cox D E, Capogna L, Cheong S-W and Marezio M 1999 Phys. Rev. B 5914440

[27] Ibarra M R, De Teresa J M, Blasco J, Algarabel P A, Marquina C, Garcia J, Stankiewicz J and Ritter C 1997 Phys. Rev. B 568252

[28] Jo Y, Park J-G, Hong C S, Hur N H and Ri H C 2001 Phys. Rev. B 63172413

[29] Kittel C 1978 Introduction to Solid State Physics (Moscow: Nauka)

[30] Causa M T et al 1998 Phys. Rev. B 583233

[31] Jaime M, Salomon M B, Rubinstein M, Treece R E, Horwitz J S and Chrisey D B 1996 Phys. Rev. B 5411914

[32] Schuddinck W, Van Tendeloo G, Barnabe A, Hervieu M and Raveau B 2000 J. Magn. Magn. Mater. 211105 